\title{
AVANCES DE LA TECNOLOGÍA DE INFORMACIÓN EN EL SECTOR PÚBLICO
}

\section{TECHNOLOGY INFORMATION ADVANCE IN THE PUBLIC SECTOR}

\section{Carlos Pastor Carrasco*}

Docente Asociado de la Facultad de Ciencias Contables, UNMSM

[Recepción: Enero de 2010 / Conformidad: Marzo de 2010]

\section{RESUMEN}

La finalidad del presente artículo es analizar las labores del gobierno en el proceso de Modernización de los Sistemas de Administración, poniendo énfasis en las tecnologías de información, el cual con el apoyo de los elementos tecnológicos que intervienen en el proceso y profesionales contables en las organizaciones gubernamentales, generarán un beneficio a la administración gubernamental para alcanzar sus objetivos. En la administración financiera del sector público, uno los factores que inciden en forma negativa es su falta de información confiable y oportuna sobre el avance y el resultado final de las operaciones económicas y financieras, debido, entre otros factores, al gran volumen de operaciones que se realiza diariamente y a los procesos de registro que genera su ejecución. A fin de superar esta problemática, los Gobiernos de América Latina vienen desarrollando esfuerzos orientados para modernizar los sistemas de administración financiera con capacidad para generar información oportuna, precisa, confiable y eficiente sobre el resultado de las actividades vitales.

Palabras clave: Modernización de la gestión del Estado, tecnologías de información, desarrollo profesional, formación académica, productividad.

\begin{abstract}
The purpose of this paper it's analyze the work of government in the process of Modernization Management Systems, with emphasis on Information Technology, which is supported by the technological elements involved in the process and accounting professionals in organizations government, generating a profit to the Government Administration to achieve its objectives. In the public sector financial management, a factor negatively affecting its lack of reliable and timely information about progress and the final result of the economic and financial operations, to other factors, the large volume of transactions occurs daily and registration processes generated by its implementation. To overcome this problem the governments of Latin America, have been developing efforts to modernize financial management systems capable of generating timely, accurate, reliable and efficient as the result of the vital activities.
\end{abstract}

Key words: Modernization of state management, information technology, professional development, academic achievement, productivity.

* Contador Público Colegiado Certificado. Docente Asociado de la Facultad de Ciencias Contables, UNMSM. Docente Investigador de la Facultad de Ciencias Contables, UNMSM. E-mail: cpc5637@hotmail.com 


\section{INTRODUCCIÓN}

En la administración financiera del sector público de los Gobiernos de América Latina, uno los factores que inciden en forma negativa en su correcto funcionamiento, es la falta de información confiable y oportuna sobre el avance y el resultado final de las operaciones económicas y financieras, debido principalmente al gran volumen de operaciones que se realiza diariamente, en cada una de las instituciones confortantes del sector público y a los procesos de registro que genera dicha ejecución.

A fin de superar esta problemática, la mayoría de los gobiernos de América Latina, vienen desarrollando esfuerzos orientados a alcanzar la modernización de los sistemas de administración financiera con una capacidad que le permita generar información oportuna, precisa, confiable y eficiente sobre el resultado de las actividades vitales.

En el Perú, dicho proceso se inicia el año 1997, cuando el Ministerio de Economía y Finanzas de esa época expidió la Resolución Viceministerial N.o 005-97-EF/11 para que en una primera etapa se inicien las pruebas de un incipiente sistema SIAF, el mismo que funcionaría en paralelo a los sistemas existentes.

Posteriormente, el Gobierno del Perú, con la finalidad de consolidar el proceso de modernización y extenderlo a todas las instituciones del Estado aprobó, en el año 2002, la Ley N.o 27658 "Ley Marco de Modernización de la Gestión del Estado" durante el gobierno del Presidente Constitucional Alejandro Toledo. En dicha ley se declara al Estado peruano en proceso de modernización en sus diferentes instancias, dependencias, entidades, organizaciones y procedimientos, con la finalidad de mejorar la gestión pública y contribuir en el fortalecimiento de un Es- tado moderno, descentralizado y con mayor participación del ciudadano.

Correspondía a la Presidencia del Consejo de Ministros coordinar esfuerzos intersectoriales para efecto de maximizar los resultados de la gestión pública motivo por el cual se expide el Decreto Supremo N.o 066-2003PCM asignando a la Oficina Nacional de Gobierno e Informática ONGI, como ente rector del Sistema Nacional de Informática.

En la actualidad, se encuentran operando o tienen proyectado ejecutar proyectos de Sistemas Integrados de Administración Financiera (SIAF) en Bolivia, Ecuador, Colombia, Venezuela, Uruguay, Argentina, Panamá, Guatemala, Nicaragua, Honduras, El Salvador, Costa Rica y República Dominicana.

Dichos Sistemas Integrados de Administración Financiera (SIAF) proporcionan información oportuna, de calidad y de buena cobertura a través de equipos de computación, mediante mecanismos que permiten el almacenamiento de información en una base de datos organizada en archivos identificables por medio de los cuales se logró una racional administración de la información evitando de registro.

\section{PLANIFICACIÓN ESTRATÉGICA}

A fin de determinar la dirección hacia donde se dirige la Administración Financiera de las empresas del sector Público es importante responder a las siguientes preguntas: ¿De dónde vienen? ¿Dónde están? ¿Hacia adónde van?

\section{¿De dónde vienen?}

El modelo tradicional de la administración pública sitúa al ciudadano ante un proceso de gran complejidad, donde debía lidiar con 
entidades extremadamente funcionales, con un control excesivo, visiones indefinidas, centralización excesiva y una burocracia ineficiente.

Donde no existían procedimientos formales y eficientes que definieran la responsabilidad de los involucrados; lo cual creaba dependencias de las personas que manejan las operaciones.

Asimismo, existía un número excesivo de operaciones o actividades de poco valor agregado y generaban pérdidas de tiempo y, además, muchos reprocesos. Finalmente, no existían indicadores funcionales que permitieran controlar el funcionamiento de la institución.

En el ámbito de empleo de Internet, muchas instituciones solo se conformaban con tener una presencia informativa. El acceso de los ciudadanos a dichas páginas era muy limitado y los servicios que brindaban no pasaban de dar a conocer su dirección y mencionar algunos servicios importantes que prestaban.

Ante directivas presidenciales, algunas entidades gubernamentales empezaron a realizar iniciativas individuales a fin de tener una mejor presencia en Internet. Pero los procesos e inversiones que se desarrollaban en este ámbito eran desarticulados y no tenían coherencia.

Por lo expuesto, podemos concluir que la presencia del gobierno en Internet, en sus inicios, era de muy baja calidad y, además, al no existir un ente coordinador entre ellas se generaba duplicación de información en las diferentes páginas que se mostraban.

\section{¿En dónde están?}

\section{E-government orientado hacia la transparencia}

El e-government, e-gobierno o gobierno electrónico consiste en el uso de las tecnolo- gías de la información y el conocimiento en los procesos internos de gobierno y en la entrega de los productos y servicios del Estado tanto a los ciudadanos como a la industria. Muchas de las tecnologías involucradas y sus implementaciones son las mismas o similares a aquellas correspondientes al sector privado del comercio electrónico (o e-business), mientras que otras son específicas o únicas en relación a las necesidades del gobierno.

Se basa principalmente en la implantación de herramientas como portales, ERPs, que en caso de los gobiernos se conocen como GRPs, CRMs, y muchas otras, buscando una mejora en la eficiencia y eficacia de los procesos internos y de vinculación con la sociedad.

Asimismo, mediante Ley N.o 27806, que aprueba la Ley de Transparencia y Acceso a la Información Pública, establece en su Art. 5 la publicación en los portales de las dependencias públicas. Las entidades de la administración pública establecerán progresivamente, de acuerdo a su presupuesto, la difusión a través de Internet de la siguiente información:

1. Datos generales de la entidad de la administración pública que incluyan principalmente las disposiciones y comunicados emitidos, su organización, organigrama y procedimientos.

2. Las adquisiciones de bienes y servicios que realicen. La publicación incluirá el detalle de los montos comprometidos, los proveedores la cantidad y calidad de bienes y servicios adquiridos.

3. La información adicional que la entidad considere pertinente.

\section{Masificación del acceso a tecnologías}

El Primer Ministro, mediante D.S. N.o 0662001-PCM, aprueba los "Lineamientos de 
Políticas Generales para promover la masificación del acceso a Internet en el Perú", en la que se establecen las acciones destinadas a lograr la masificación del acceso a Internet en el Perú, deben considerar entre otros aspectos los siguientes:

1. Promover la masificación del acceso y uso de Internet y las tecnologías de la información, optimizando y desarrollando la infraestructura de telecomunicaciones existente a nivel nacional.

2. Promover la expansión del acceso a Internet a nivel nacional, a usuarios domiciliarios, o a través de cabinas públicas de acceso a Internet. Asimismo, fortalecer los planes gubernamentales que permitan el acceso a Internet en zonas rurales y de preferente interés social.

3. Las entidades de la administración pública deberán incluir en sus planes sectoriales, así como en el desarrollo de sus actividades, metas relacionadas con el uso de Internet y el uso de herramientas informáticas, a fin de agilizar la prestación de servicios gubernamentales y propender a la prestación de servicios en línea (gobierno electrónico) a través de páginas web y servicios de consulta interactivo.

4. El Ministerio de Educación en coordinación con los organismos correspondientes, deberá realizar, entre otros, las siguientes acciones:

- Presentar anualmente planes para la dotación del servicio de acceso a Internet en los centros educativos, bibliotecas públicas y universidades, estableciendo metas para tal efecto, ello deberá incluir la dotación de herramientas informáticas de software $y$ hardware de acceso a Internet.

- En un plazo no mayor de dos (2) años deberán estar disponibles en Internet, programas educativos, recursos di- dácticos y material de consulta desarrollados en base a la currícula educativa, a fin de promover su utilización masiva. Para ello, se deberá realizar un inventario de los programas, recursos didácticos y herramientas informáticas que están siendo utilizados por las universidades y otras instituciones educativas a nivel de América Latina para educación a distancia, con el fin de evitar duplicidad de esfuerzos.

- Crear planes de capacitación para la población en general sobre el uso y beneficios del Internet, y el manejo de herramientas informáticas, a fin de promover una cultura de auto aprendizaje y capacitación a distancia.

5. Promover la reducción de costos en todos los componentes que permitan la conexión a Internet por parte de las entidades competentes, en particular de los que constituyen herramientas indispensables para la masificación del acceso a Internet.

\section{Estrategia institucional, Estándares y Políticas}

Mediante Ley N.o 27658, Ley Marco de Modernización de la Gestión del Estado, se declara al Estado peruano en proceso de modernización en sus diferentes instancias, dependencias, entidades, organizaciones y procedimientos, con la finalidad de mejorar la gestión pública y contribuir en el fortalecimiento de un Estado moderno, descentralizado y con mayor participación del ciudadano.

El Estado peruano ha dado pasos importantes para el desarrollo del gobierno electrónico, los que deberán de ser articulados para 
efectos de maximizar los resultados de las distintas instancias comprometidas en dicho esfuerzo, mejorar su gestión y optimizar la atención al ciudadano.

La Presidencia del Consejo de Ministros es la instancia encargada de coordinar esfuerzos intersectoriales para efectos de maximizar los resultados en la gestión pública; que, de conformidad con el Decreto Supremo N. ${ }^{\circ}$ 066-2003-PCM y el artículo 34 del Decreto Supremo N. ${ }^{\circ}$ 094-2005-PCM, Reglamento de Organización y Funciones de la Presidencia del Consejo de Ministros, la Secretaría de Gestión Pública actúa como ente rector del Sistema Nacional de Informática.

En cumplimiento a lo dispuesto por el artículo $37^{\circ}$ del Reglamento de Organización y Funciones de la Presidencia del Consejo de Ministros, la Oficina Nacional de Gobierno Electrónico e Informática - ONGEI de la Presidencia del Consejo de Ministros ha presentado la Estrategia Nacional de Gobierno Electrónico e Informática;

La Estrategia Nacional de Gobierno Electrónico es una meta incluida en la matriz del Plan de Desarrollo de la Sociedad de la Información en el Perú - La Agenda Digital Peruana, aprobada mediante Decreto Supremo N. ${ }^{\circ}$ 031-2006-PCM. Motivo por el cual se establece una Estrategia Nacional de Gobierno Electrónico, que constituye un instrumento de gestión para definir las actividades informáticas de las entidades de la administración pública integrantes del Sistema Nacional de Informática en sus diferentes niveles y coordinar los esfuerzos de las entidades de la administración pública.

\section{Presencia de Internet}

El Gobierno del Perú y las instituciones públicas han puesto mucho de su parte para crear sitios en internet y facilitar a los ciudadanos un acceso en línea a la información de la administración pública (Ejemplo como la SUNAT, Ministerios, Indecopi, CONASEV, Portal del Gobierno Peruano en línea y otros, son pasos fundamentales). Los mejores sitios son aquellos que responden a las necesidades de los usuarios no dotados de conocimientos técnicos y que permiten acceder a un gran abanico de información jurídica y administrativa. Pero hace falta hacer más para que los sitios Internet sean herramientas de fácil utilización para obtener información y comunicarse con los distintos servicios de la administración pública, observando siempre las normas legales sobre protección de datos.

El objetivo de la acción es facilitar el acceso a la información del sector público extendiendo y simplificando el acceso a Internet. De este modo se incentivará el desarrollo de nuevos servicios del sector privado basados en las nuevas fuentes de datos que sean accesibles.

\section{Inversión}

Destacados economistas -Paul Romer, Robert Lucas, Robert Solow y Frank Ramsey, entre otros- han explicado cómo la inversión en investigación y desarrollo (I+D) tiene efectos positivos sobre el crecimiento económico. Por ejemplo, hace cuatro decenios Solow (1956) demostró que solo las mejoras tecnológicas son capaces de generar un crecimiento sostenido en el largo plazo. Investigaciones recientes han señalado que las inversiones en investigación y desarrollo orientadas hacia las innovaciones tecnológicas contribuyen al crecimiento económico al crear conocimientos y prácticas que tienen externalidades positivas significativas, mejo- 
ran los procesos productivos y contribuyen a elevar los niveles de vida.

Por otro lado, diversos estudios indican que la rentabilidad de la inversión en innovación, ciencia y tecnología es muy alta. Las tasas de retorno de este tipo de inversión son, en general, de 30 a $80 \%$ mientras que las tasas de rentabilidad social superan el $60 \%$. Además, las tasas de retorno a las inversiones en investigación científica y tecnológica agropecuaria alcanzan del 40 a $60 \%$ por año.

En resumen, el conocimiento científico, el desarrollo tecnológico y la innovación son la clave para aumentar la productividad y competitividad, reducir la pobreza, conservar el medio ambiente y usar sustentablemente los recursos naturales, mejorar la calidad de vida y lograr la plena realización de las personas.

\section{¿Hacia adónde van?}

Dentro de las acciones del Gobierno, podemos mencionar a las siguientes:

- Constitución de empresas en línea

- Ventanilla Única de Servicios al Ciudadano y la Empresa (Integración SUNARP, SUNAT, RENIEC y Notarios).

- Ventanilla Única de Comercio Exterior.

- Integración electrónica de exportadores, importadores y operadores de comercio exterior con las entidades del Estado que participan en comercio exterior.

- Planillas electrónicas.

- Integración electrónica de empleadores con la SUNAT, ESSALUD y el Ministerio de Trabajo.

- Infraestructura Oficial de Firmas Digitales del Estado Peruano.

- Implementación de la Autoridad Administrativa Competente y la Infraestructura Oficial de firmas por parte del RENIEC.
- DNI electrónico.

- Implementación del documento de identidad electrónico por parte de RENIEC

- Factura Electrónica.

- Integración electrónica de los contribuyentes con la SUNAT.

- Libros contables electrónicos.

- Integración electrónica de contribuyentes con la SUNAT.

\section{SISTEMA NACIONAL DE PRESUPUESTO}

La Gestión presupuestal se basa en la siguiente normatividad:

- Constitución Política del Estado peruano.

- Ley N.o 28112, Ley Marco de la Administración Financiera del Estado.

- Ley N.o 28411, Ley General del Sistema Nacional de Presupuesto.

- Ley N.o 29289, Ley del Presupuesto del Sector Público para el Año Fiscal 2009.

- Directivas e instructivo del proceso presupuestario emitido por la DNPP y Fondo Nacional de Financiamiento de la Actividad Empresarial del Estado.

Actualmente la Gestión Presupuestaria tiene la necesidad de:

a) Reducir la inercia presupuestaria vinculando asignaciones a incrementos en productos, servicios y resultados.

b) Articular la planificación estratégica con la programación presupuestaria.

c) Generar incentivos en el proceso presupuestario a una gestión eficaz.

d) Desarrollar evaluaciones efectivas que generen información adecuada para la toma de decisiones.

e) Lograr que el presupuesto público sea una herramienta real de gestión que incida en la eficacia y eficiencia de la acción 
en cumplimiento de las funciones del Estado.

\section{Visión integral del sistema de planeamiento}

El Sistema Nacional de Presupuesto debe ser visto como una Visión Integral que involucra en principio una Visión Nacional que conforma la Imagen Objetivo o Agenda estratégica del Gobierno. Las políticas del Estado deben estar en el concepto de la nacional, la política general de gobierno de incluirse en los programas y posiciones. El marco multianual debe generarse como consecuencia de la política general de gobierno y debe incluir proyecciones y recomendaciones, los lineamientos de política sectorial y el plan de desarrollo concertados deben de ejecutarse a nivel territorial. Cada institución de gobierno debe elaborar un plan estratégico institucional, y como elemento de gestión debe elaborarse el presupuesto anual.

De seguirse de acuerdo a esta visión, el presupuesto se convierte en un instrumento básico en la obtención de los objetivos nacionales.
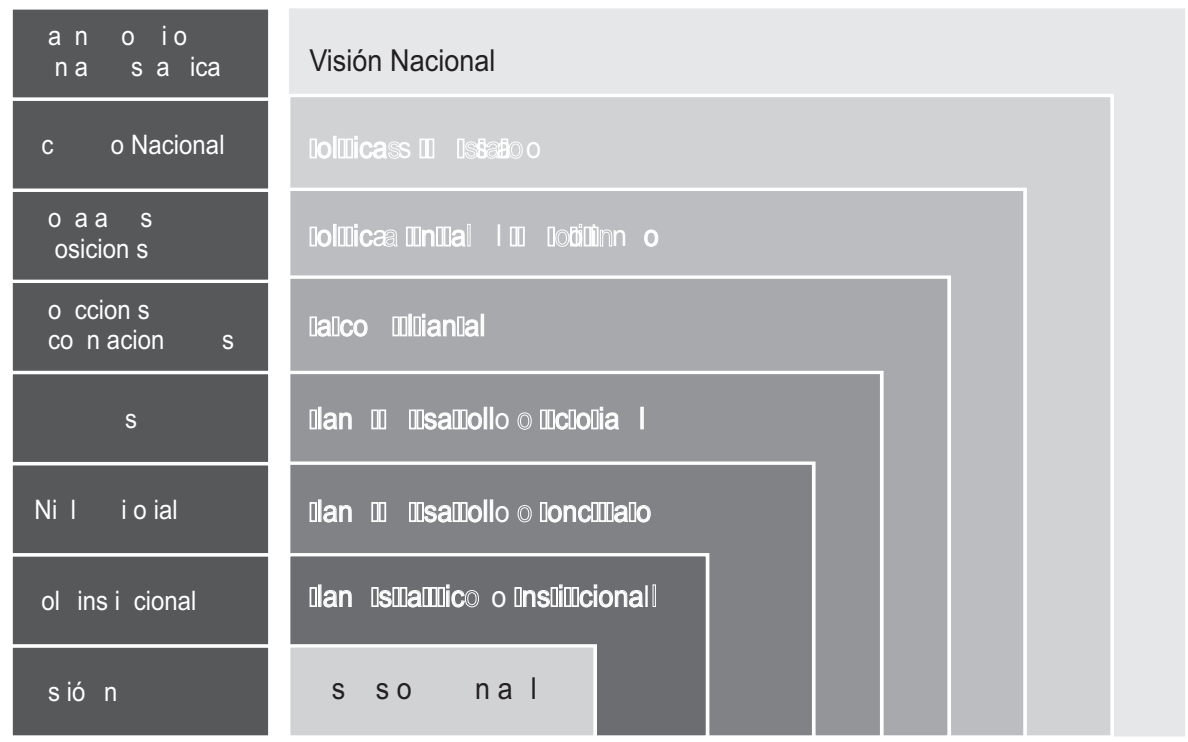

Ilustración N.o 1. Visión integral del sistema de planeamiento.

\section{Flujograma de actividades del proceso presupuestario y sus limitaciones}

Del seguimiento de las actividades del proceso presupuestario se han podido determinar que existe en las siguientes limitaciones:

- Reducida o nula incidencia en asignación institucional y discusión pública.

- Presupuesto incrementar, sobre la base de los históricos.
- Estructura de presupuestación rígida, no dan cuenta de productos, resultados ni costos.

- Discrecionalidad en el nivel político.

- Reducida incidencia del poder legislativo.

- Evaluaciones que no retroalimentan los procesos de toma de decisiones.

Todos los aspectos antes mencionados originaron que el presupuesto público debía 
cambiar su estructura y proceso de aprobación.

\section{Gestión presupuestaria por resultados}

En el marco de la corriente de Nueva Gerencia Pública o New Public Management, propone un cambio significativo en la presupuestación orientando el foco de atención hacia los resultados de la acción y la gerencia pública.

Su implementación plena requiere el desarrollo de los siguientes elementos:

- Estructura de programas de gasto compatibles con el Planeamiento Estratégico.

- Rediseño (de ser necesario) de estructuras programáticas existentes, especificando productos (bienes y servicios), costos y resultados asociados.

- Nuevo esquema de incentivos y de gerencia pública ("dejar gerenciar").

- Nuevo esquema de rendición de cuentas y accountability (vigilancia social).

- Seguimiento y evaluación que de cuenta de productos, resultados e impactos.

\section{SISTEMA INTEGRADO DE INFORMACIÓN FINANCIERA}

\section{Concepto}

El Sistema Integrado de Administración Financiera (Denominado de ahora en adelante SIAF) es un sistema de proceso de operaciones desarrollado por el Ministerio de Economía y Finanzas (MEF), que automatiza la información de los procesos de ejecución presupuestaria y financiera mediante un registro único de operaciones produciendo con mayor rapidez, precisión e integridad informes utilizados para una adecuada toma estructura de decisiones (Estratégicas, tácticas y opera- tivas) por los diferentes niveles gerenciales de la organización gubernamental.

Es un sistema de Registro Único que permite realizar:

- La formulación del presupuesto, sus modificaciones y la asignación trimestral.

- La ejecución de los gastos e ingresos.

- La gestión de pagaduría a proveedores (incluyendo cobranza coactiva y servidores públicos).

- La contabilización, de operaciones y la elaboración de EEFF y anexos.

- El control de la deuda interna y externa.

- El control del pago de planilla y de los servicios no personales.

- El seguimiento a través de consultas y reportes.

No contempla:

- Administración de las personas

- Procesos logísticos (abastecimiento y de control patrimonial)

\section{Ámbito}

El ámbito comprende los siguientes sistemas:

a) Sistema asociado a la ejecución del presupuesto anual

b) Sistema de registro único de operaciones de gastos e ingresos presupuestarios

c) Sistema que integra la información de los proceso de ejecución presupuestaria, financiera y contable.

El SIAF vincula diferentes instituciones:

- Organismos rectores del MEF (DNPP, DGTP, CPN).

- Organismos sectoriales (pliegos, unidades gestoras y unidades ejecutoras).

La unidad ejecutora es la unidad orgánica de referencia del SIAF, con capacidad para desarrollar funciones administrativas y contables. Depende de las Unidades Gestoras (UGEST). 


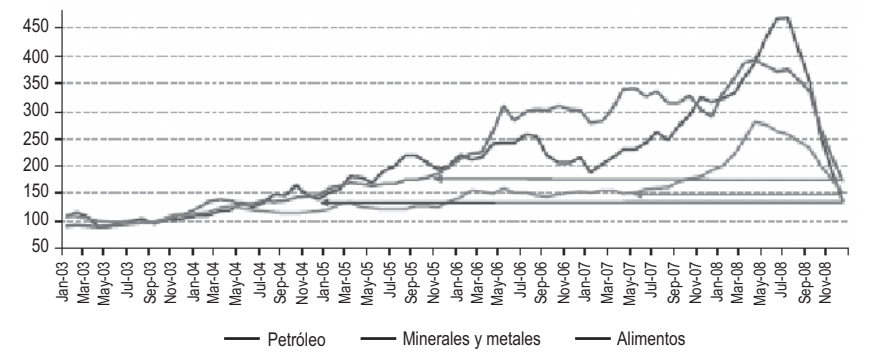

Ilustración N.o 2. Sistema Integrado de Administración Financiera del Sector Público

\section{Objetivos}

En general, el SIAF tiene los siguientes objetivos:

- Registrar en forma integral todas las operaciones que se producen en los organismos públicos y que afectaban la gestión presupuestaria y financiera de la administración gubernamental.

- Presentar información contable adecuada y oportuna con el nivel de detalle suficiente sobre el resultado de las operaciones financieras y presupuestarias realizadas por entidades del sector público.

- Fortalecer las labores del Control Interno Gerencial.

- Suministrar información para la adecuada toma de decisiones por parte de los responsables de la gestión financiera gubernamental.

- Facilitar la auditoría interna y externa.

- Contribuir a mejorar la gerencia de la administración financiera gubernamental.

\section{Ventajas}

Dentro de las ventajas que se obtiene de implementar el SIAF en las entidades gubernamentales, podemos mencionar las siguientes:

- Proporcionar a sus diferentes usuarios información oportuna, de calidad y de buena cobertura.

- Permitir reportes consistentes de estados presupuestales, financieros y contables.

- Permitir un seguimiento de la ejecución presupuestaria en sus diferentes fases.

- Proporcionar una visión global y permanente de la disponibilidad de los recursos financieros de cada entidad o del Estado.

- Contribuir a una mejor asignación de los recursos financieros y toma de decisiones.

- Registro obligatorio (cobertura 100\%).

- Información oportuna y consistente.

- Seguimiento de la ejecución presupuestaria en sus diferentes fases.

- Visión global y permanente de la disponibilidad de recursos financieros del Estado. 
- Contribuye en la mejor asignación de los recursos y toma de decisiones.

\section{Registros}

El registro de las operaciones presupuestarias en el SIAF se inició en enero de 1997, tal como se desprende de la Resolución Viceministerial N. ${ }^{\circ} 005-97-E F / 11$. En una primera etapa, mientras se realizaban las pruebas y alcance de la cobertura adecuada, el SIAF se registraba en paralelo a los ya existentes.

Posteriormente, mediante la Ley $\mathrm{N}$. 28112, Ley Marco de la Administración Financiera del Sector Público (Ley SAFI) se establece lo siguiente:

- Articulo $10^{\circ}$. Registro Único de Información.
10.1. El registro de información es único y de uso obligatorio por parte de todas la entidades y organismos del sector público, a nivel nacional, regional y local y se efectúa a través del Sistema Integrado de Administración Financiera del Sector Público (SIAF-SP) que administra el Ministerio de Economía y Finanzas, a través del Comité de Coordinación.

10.2. El SIAF-SP constituye el medio oficial para el registro, procesamiento y generación de la información relacionada con la administración financiera del sector público, cuyo funcionamiento y operatividad se desarrollan en el marco de la normatividad aprobada por los órganos rectores.

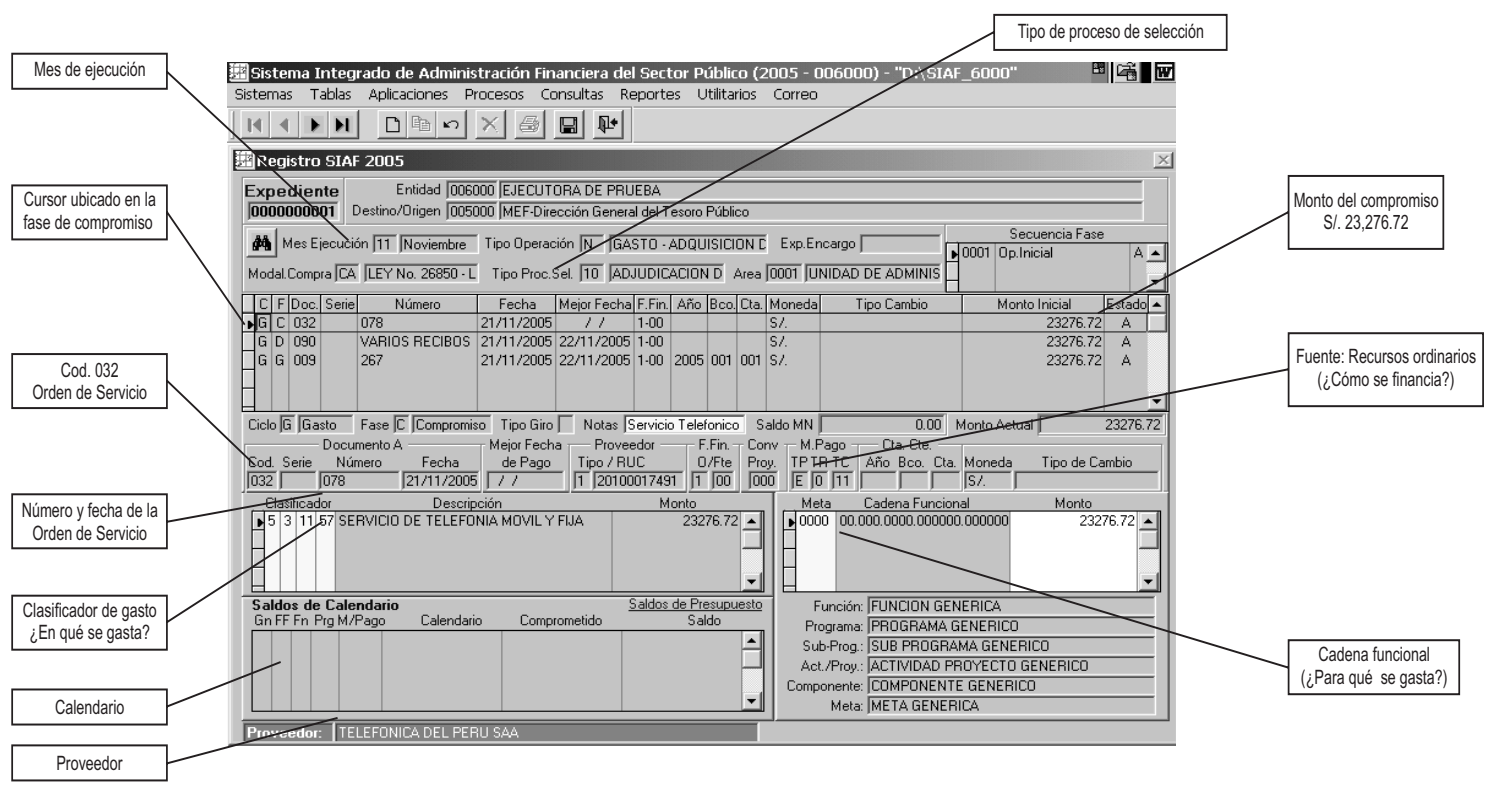

Ilustración N.o 3. Pantalla de Registro SIAF. 
Reportes que proporciona el Módulo SIAF-SP

Los tipos de reportes proporcionados por el modulo SIAF-SP son:

a) Reportes SIAF

Presentan información del ejecución presupuestaria y financiera, a diferentes niveles de agregación: por mes, por operación, por meta, por Programa/Grupo Genérico, por Clasificador de Gastos.

Estos reportes, además de contar con opciones de filtro (por registro, rango de registros, o fase, Tipo de Operación, Fuente de Financiamiento y Estado de Envío), pueden ser exportados a una Hoja de Excel, donde la posibilidad de filtro y ordenamiento son múltiples.

b) Reportes Presupuestales

Permiten el seguimiento de la ejecución de los compromisos frente al calendario y, además conocer la recaudación ingresos. El Reporte Ejecución del Gasto (Detallado) presenta la información de la ejecución del gasto a nivel de Grupo Genérico, en sus fases Comprometido, Devengado y Girado, mostrando de forma discriminada las modificaciones realizadas (Ampliaciones, Rebajas, Devoluciones, Anulaciones) en la Operación Inicial.

El módulo SIAF-SP permite realizar consultas de la ejecución de ingresos, de los documentos ingresados como sustento de una fase, es decir, permite conocer cuánto se recaudó durante el año; ingresando Menú Reportes de los sub-módulos: SIAF-Resumen de Recaudación Ingresos.

c) Reportes de Tesorería

Presentan información de la ejecución financiera.

El Informe de Devengados Pendientes de giro (T-1) presenta información de los gastos devengados, pendiente de giro.
El Informe de Ingresos por Fuente (T-5), permite apreciar los montos de recaudación realizados por la UE a nivel de Específica de Ingresos (3 dígitos).

El Informe Devoluciones por Encargo presenta información de las operaciones de Encargo otorgadas, que cuentan con saldos para ser reprogramados por el Tesoro Público.

Presente listado de Comprobantes de Pago de acuerdo en mes y tipo de operación solicitada.

d) Reportes Contables

Utilizados en el sistema de contabilidad gubernamental, que van de los libros contables principales (libro diario, libro mayor) y auxiliares (libro bancos) a los estados financieros y presupuestarios.

El menú reportes contables también comprende el Plan Contable Gubernamental.

e) Reportes SUNAT

Muestra la información registrada para SUNAT para la confrontación de operaciones autodeclaradas (COA). El reporte generado por el Módulo se denomina Registro COA.

f) Reporte de Maestros

Comprende los siguientes reportes: Cadena funcional, cadena institucional, clasificadores del gasto, clasificador en ingresos, finalidad.

\section{CONCLUSIONES}

1. El registro adecuado y oportuno de las Transacciones Financieras del Estado hace que este sea integrado y optimizado, obtenga una adecuada transparencia en la gestión pública y le permita enfrentar proyectos de TI con ROI claros y efectivos, asimismo se obtenga una 
adecuada confianza ciudadana sobre el accionar del Estado.

2. Al integrar las TIC a las actividades del Gobierno, permite la utilización de mecanismos de comercio electrónico para dinamizar la economía en temas de e-goverment las inversiones son bajas frente a su retorno.

3. Migrar de:

a) Un modelo de gestión de TI orientado a servicios.

b) A un modelo de TI enfocado en la gestión de Información, generando una adecuada Participación estratégica de TI.

4. El uso integrado de las TIC en el manejo gubernamental y en especial en el SIAF:

a) Se genera un sistema que asegure la preservación y el respeto a la información personal e institucional.

b) Se genera una cultura ética de gestión transparente, donde toda la información pertinente sea de acceso público.

c) Se crea un sistema de información confiable y oportuna para una efectiva toma de decisiones en el Gobierno.

d) Se consigue un ahorro en las operaciones del gobierno.

e) Se genera una cultura de servicio basada en las herramientas que brinda la Sociedad de la Información. Se acerca el Gobierno, al ciudadano y a la empresa con servicios de calidad, modernos, eficientes y confiables disponibles las 24 horas, los 365 días al año.

5. En el Perú, el gobierno electrónico está en vías de desarrollo; sin embargo, parece cada día más evidente la existencia de una tendencia nacional hacia su implementación.

\section{REFERENCIAS BIBLIOGRÁFICAS}

1. Aghion, P. y Howitt, P. (1998). Endogenous growth theory. Cambridge, MA: MIT Press.

2. CODESI (2006). Estrategia nacional de gobierno electrónico. [web: http:// www.codesi.gob.pe/codesi/downloads/ENGEI.pdf.]. Fecha de consulta: 10/10/2009, Lima.

3. Huidobro, J. y Roldán D. (2005). La tecnología e-business. PARANINFO, España.

4. Laurence Wolff (1999). "Acceso de alta velocidad al internet: El futuro del mundo y sus implicaciones para los países en desarrollo". Scientific American.

5. Solow, R. (1956), "A contribution to the theory of economic growth". MA: Quarterly Journal of Economics. 